\title{
Transvaginal Ultrasound
}

National Cancer Institute

\section{Source}

National Cancer Institute. Transvaginal Ultrasound. NCI Thesaurus. Code C17644.

A technique in which sound waves are sent out by an ultrasound probe that has been inserted in the vagina and a computer uses the ultrasound echoes to create an image of the female genital tract. The test can be performed to evaluate women with infertility problems, abnormal bleeding, sources of unexplained pain, congenital malformations of the uterus and ovaries, and possible tumors and infection. 